\title{
Crystalline chirality and interlocked double hourglass Weyl fermion in polyhedra-intercalated transition metal dichalcogenides
}

\author{
Pu Huang ${ }^{1}$, Xinbo Chen ${ }^{1}$, Peng Zhang ${ }^{1}$, Hongyi Sun ${ }^{2}$, Shaogang $X^{1}{ }^{1}$, Wen Xiong ${ }^{3}$, Rui Wang $\mathbb{1}^{3}$, Han Zhang $\mathbb{1}^{4}$, \\ Qihang Liu $\mathbb{D}^{2}$ and Xiuwen Zhang (1)
}

\begin{abstract}
Introducing crystalline chirality into transition metal dichalcogenides (TMDs) has attracted much attention due to its modulation effect on optical properties and the potential to reveal new forms of electronic states. Here, we predict a number of chiral materials by intercalating polyhedra into TMD lattices, finding a type of double hourglass Weyl fermion interlocked with crystalline chirality. The best candidate $\mathrm{RhV}_{3} \mathrm{~S}_{6}\left(\mathrm{PG}_{3} 22\right)$ possesses the largest hourglass energy window of $\sim 380 \mathrm{meV}$, as well as strong optical circular dichroism (CD) in the infrared regime, both of which are tunable by external strains. The chirality is originally induced by the configuration of intercalated polyhedra and then reduced by the rotational atomic displacements triggered by intercalation, as indicated by CD calculations. Our study opens the way of designing chiral materials with spin-split double hourglass Weyl fermions via structural unit intercalation in achiral crystals for future chiral-functionalized optoelectronic and spintronic devices.
\end{abstract}

\section{Introduction}

Transition metal dichalcogenides (TMDs) are the subject of intense interest due to their remarkable electronic and optical properties, such as their direct bandgap in a monolayer limit ${ }^{1}$, strongly bound excitons and trions ${ }^{2,3}$, and chiral optical selection rules $^{4,5}$ due to spin-valley locking degrees of freedom. Benefitting from these unique properties, especially the chiral selection rules, TMDs have acted as an ideal class of materials for optoelectronic and spintronic manipulation, becoming increasingly studied for valleytronic devices ${ }^{6}$. As the chiral optical selectivity ${ }^{4,5}$ of the $K$ and $K^{\prime}$ valleys have

Correspondence: Rui Wang (rcwang@cqu.edu.cn) or Han Zhang (hzhang@szu. edu.cn) or Qihang Liu (liugh@sustech.edu.cn) or

Xiuwen Zhang (xiuwenzhang@szu.edu.cn)

${ }^{1}$ Key Laboratory of Optoelectronic Devices and Systems of Ministry of Education and Guangdong Province, College of Physics and Optoelectronic Engineering, Shenzhen University, 518060 Shenzhen, China

${ }^{2}$ Shenzhen Institute for Quantum Science and Technology and Department of

Physics, Southern University of Science and Technology (SUSTech), 518055

Shenzhen, China

Full list of author information is available at the end of the article opposite signs in TMDs without crystalline chirality, external chirality has been applied to tune the valleytronic property of $\mathrm{TMDs}^{7-10}$. Valley polarized photoluminescence of TMDs such as $\mathrm{MoS}_{2}$ can be tailored through nearfield interactions with plasmonic chiral metasurfaces ${ }^{8}$. The coupling of valley excitons in monolayer $\mathrm{WS}_{2}$ with chiral surface plasmons has also been demonstrated ${ }^{10}$, which occurs at room temperature and persists for a long lifetime. Most recently, external crystalline chirality was induced in $\mathrm{MoS}_{2}$ nanostructures via the surface modification of chiral ligands ${ }^{11,12}$. The coupling of external chirality with intrinsic chiral optical selectivity significantly enriches the phenomena in the TMD material family.

Weyl fermions in TMDs ${ }^{13-17}$ have also attracted much attention in light of their extremely large magnetoresistance for potential applications in magnetic sensors and memory ${ }^{18,19}$. The Weyl points in these achiral TMD Weyl semimetals $^{13-15}$ are paired with opposite chirality. Therefore, coupling external net chirality to the chirality of Weyl fermions could further advance the study of

\section{(c) The Author(s) 2021}

(c) (i) Open Access This article is licensed under a Creative Commons Attribution 4.0 International License, which permits use, sharing, adaptation, distribution and reproduction cc) in any medium or format, as long as you give appropriate credit to the original author(s) and the source, provide a link to the Creative Commons license, and indicate if changes were made. The images or other third party material in this article are included in the article's Creative Commons license, unless indicated otherwise in a credit line to the material. If material is not included in the article's Creative Commons license and your intended use is not permitted by statutory regulation or exceeds the permitted use, you will need to obtain permission directly from the copyright holder. To view a copy of this license, visit http://creativecommons.org/licenses/by/4.0/. 
TMD-based materials and potentially open the way of finding new fermions, such as the Kramers-Weyl fermions $^{20}$ that are commonly found in nonmagnetic chiral crystals when Kramers theorem-forced band degeneracy appears near the Fermi level $\left(\mathrm{E}_{\mathrm{F}}\right)$. The recently found new form of fermion, namely, the hourglass fermion ${ }^{21-24}$, is also closely related to the Weyl fermion, which, however, has not yet been found in TMDs. This class of new fermions was originally predicted on the surface of nonsymmorphic crystal $\mathrm{KHgSb}^{22}$ and then widely found in the electronic structures of nonsymmorphic materials, including three-dimensional $\mathrm{ReO}_{2}{ }^{25}, \mathrm{Al}_{3} \mathrm{FeSi}_{2}{ }^{26}$, $\mathrm{Ag}_{2} \mathrm{BiO}_{3}{ }^{27,28}, \mathrm{AgF}_{2}{ }^{29}$, and $\mathrm{Tl}_{3} \mathrm{PbBr}_{5}{ }^{30}$, as well as the hypothetical two-dimensional structure $\mathrm{Bi} / \mathrm{Cl}-\mathrm{SiC}(111)^{31}$. The predicted hourglass fermion on the surface of $\mathrm{KHgSb}^{22}$ has been observed in experiments via angleresolved photoemission spectroscopy ${ }^{32}$. However, this type of hourglass-like electronic structure usually appears as single sets. Pairs of hourglass Weyl fermionic electronic structures have not been discussed before, which is plausible, as their splitting and annihilation could be associated with a physical quantity, such as crystalline chirality.

In this paper, we search for TMD-based chiral materials with double hourglass Weyl fermions by polyhedral intercalation in a hexagonal lattice. Based on the stability and electronic property evaluation in the framework of density functional theory (DFT) ${ }^{33,34}$, we found a few intercalated chiral TMDs with pairs of hourglass fermionic electronic structures around $E_{F}$ and identified $\mathrm{RhV}_{3} \mathrm{~S}_{6}$ $\left(\mathrm{P}_{3} 22\right)$ as the best candidate with the largest hourglass window of $\sim 380 \mathrm{meV}$. The splitting and spin polarization of the double hourglass fermion was interlocked with the crystalline chirality. We evaluated the optical circular dichroism (CD) for $\mathrm{RhV}_{3} \mathrm{~S}_{6}$ and found that when the rotational atomic displacements around the chiral axis triggered by polyhedral intercalation were increased (decreased), the CD response was reduced (enhanced), suggesting that crystalline chirality was originally induced by polyhedral intercalation and then reduced by rotational atomic displacements. Furthermore, the energy window of the double hourglass fermion and optical CD could be effectively tuned by external strains. Our study opens the way for designing TMD-based materials with interlocked crystalline chirality and double hourglass Weyl fermions, with potential applications in future optoelectronic and spintronic devices.

\section{Methods}

\section{Electronic structure calculation}

All electronic structures were calculated by $\mathrm{DFT}^{35}$ as implemented in the Vienna ab initio simulation package $^{33,36}$. The projector-augmented wave pseudopotentials $^{33}$ with the exchange-correlation functional of
Perdew-Burke-Ernzerhof ${ }^{34}$ were adopted. The cutoff energy for the plane waves was selected as $520 \mathrm{eV}$, and the Brillouin zone was sampled on $10 \times 10 \times 4$ MonkhorstPack $^{37} k$-point meshes. The lattice constants and atomic positions were fully relaxed when the absolute total energy difference between two successive loops and the Hellmann-Feynman force on each atom were $<10^{-6} \mathrm{eV}$ and $5 \times 10^{-3} \mathrm{eV}^{-1}$, respectively, using the conjugate gradient algorithm. The band structure from the hybrid functional (HSE06) ${ }^{38,39}$ was obtained for comparison with the conventional DFT result. Perturbation was added to the pseudopotential to address the spin-orbit coupling (SOC) effect. The spin state of the electron wavefunction was evaluated by projecting the calculated wavefunction on the spin and orbital basis of each atomic site, which generated spin-projected band structures with dense $k$ meshes in the Brillouin zone. Strain was applied for the deformation calculation by inducing lattice shrinkage and expansion for tensile and compressive situations.

\section{Circularly polarized evaluation}

The circularly polarized optical absorption was the difference between the absorption of left- and righthanded lights for all $k$ points within the valence bands and the conduction bands, and these could be normalized and evaluated from the conventional DFT-calculated $\omega$ frequency-dependent imaginary part of the dielectric function with the random phase approximation (RPA) and Fermi golden rule ${ }^{40-42}$ :

$$
\begin{aligned}
\varepsilon_{\alpha \beta}^{(2)}(\omega)= & \frac{e^{2} h^{4}}{4 \pi^{2} \Omega \omega^{2} m_{e}^{2}} \lim _{\mathrm{q} \rightarrow 0} \sum_{c, v, k} 2 w_{\mathrm{k}} \delta\left(E_{c \mathrm{k}+\mathrm{q}}-E_{v \mathrm{k}}-\omega\right) \\
& \times\left\langle u_{c \mathrm{k}}\left|i \nabla_{a}-\mathrm{k}_{a}\right| u_{v \mathrm{k}}\right\rangle\left\langle u_{c \mathrm{k}}\left|i \nabla_{\beta}-\mathrm{k}_{\beta}\right| u_{v \mathrm{k}}\right\rangle^{*}
\end{aligned}
$$

where $\Omega$ is the volume of the primitive cell, $e$ is the elementary charge, $h$ is the Planck constant, $\omega$ represents the angular frequency, $m_{e}$ is the electron rest mass, $c$ and $v$ indicate the conduction and valence band states, respectively, $E$ is the eigenenergy of the wavefunction, $\mathbf{q}$ stands for the Bloch vector of the incident wave, $u$ is the wavefunction, $w_{\mathrm{k}}$ denotes the $k$-point weights that sum to 1 and the factor 2 before it accounts for the fact that degenerating spin has been considered, and $\nabla$ is the momentum operator, in which the sum over momentum $\boldsymbol{k}$ is conducted over the full $3 \mathrm{D}$ space. The optical $\mathrm{CD}$ is obtained by decomposing the circularly polarized part from the total absorption spectrum, as implemented in the PWmat code ${ }^{43,44}$. The circularly polarized response for $\mathrm{RhV}_{3} \mathrm{~S}_{6}$ was determined with the RPA-GGA approach. A planewave basis set was employed at a cutoff energy of $80 \mathrm{Ry}$, and a total of 212 bands were included to ensure convergence of all computed quantities. A very dense $k$-point mesh of $14 \times 14 \times 6$ over the reducible hexagonal Brillouin zone was sampled in our calculations. 


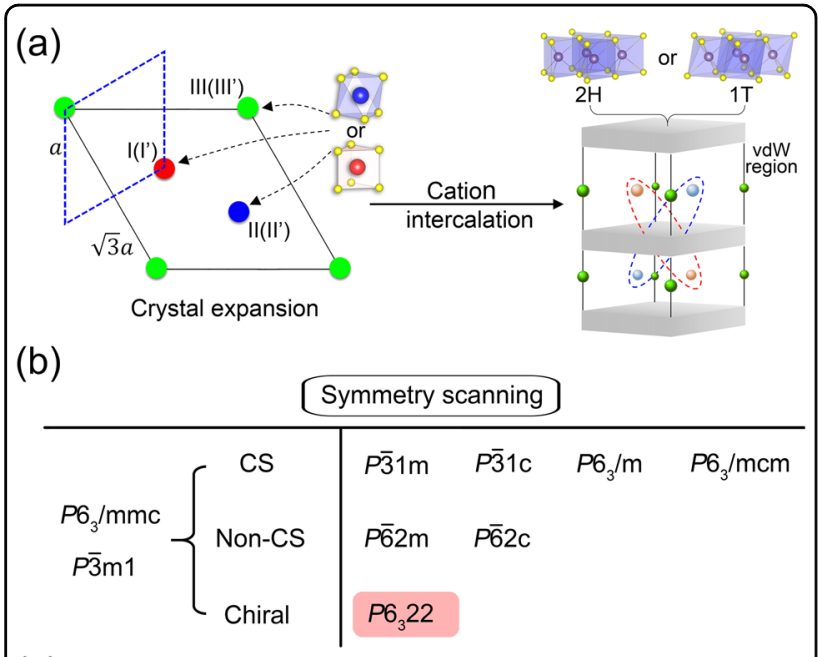

(c)

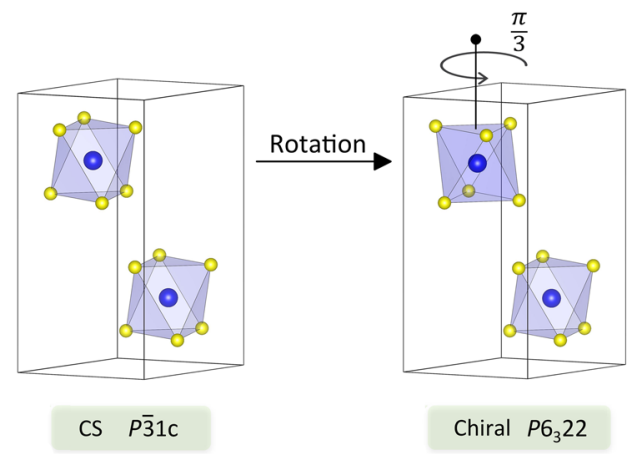

Fig. 1 Structural reconstruction and symmetry evolution of intercalated TMDs. a Crystal expansion for TMDs with ab in-plane $\sqrt{3} \times \sqrt{3}$ cell units and two $M X_{2}$ layers forming a new hexagonal lattice with six equivalent atomic sites $I\left(I^{\prime}\right), \|\left(I^{\prime}\right)$, and III(III'), which can be used to assign intercalated cations with two types of possible polyhedron motifs (octahedron and triangular prism). The gray planes represent the layered TMDs with the $2 \mathrm{H}$ or $1 \mathrm{~T}$ phase, and the colored spheres (yellow, red, and blue) within the vdW region are the intercalated cations. We denote the specific chiral intercalation patterns $\left(\mathrm{PG}_{3} 22\right)$ for $\mathrm{AB}$ stacked $2 \mathrm{H}$ TMDs with red and blue dashed ellipses. $\mathbf{b}$ Symmetry types for the polyhedra-intercalated TMDs with centrosymmetric (CS), chiral, and (achiral) non-CS SGs (see Fig. S1 for these structures). c Symmetry evolution for the hexagonal crystal filled with nonvertically distributed ideal octahedra, which indicates that only the specific arrangement of octahedra $\left(\mathrm{PG}_{3} 22\right)$ induces chirality into the system.

\section{Thermodynamic and kinetic stability assessment}

Usually, we can construct a set of candidate structures (containing their compositions) that exist in crystal databases, e.g., $\operatorname{ICSD}^{45}$ or Material Project ${ }^{46}$, compute their total energies, and then screen this list for the lowest-energy structure. To determine the thermodynamic stability of $\mathrm{AB}_{3} \mathrm{C}_{6}$ compounds, we considered all of its possible disproportionation channels to the competing phases (elemental phases, binaries and ternaries) by solving a set of inequalities in multidimensional spaces of chemical potentials $\left(\Delta \mu_{\mathrm{A}}, \Delta \mu_{\mathrm{B}}\right.$, and $\left.\Delta \mu_{\mathrm{C}}\right)$, which were presented in detail in our previous work ${ }^{47}$. The most stable cation intercalation position was confirmed through dividing the vdW planes of the layered TMDs with a dense mesh, globally detecting the symmetry evolution and total energy landscape of the system. To further evaluate the dynamic stability, we calculated the phonon dispersion curves using the finite-displacement approach as implemented in the Phonopy code ${ }^{48}$. The phonon frequency was derived from crystal forces originating from displacements of certain atoms in a $2 \times 2 \times$ 2 supercell for $\mathrm{RhV}_{3} \mathrm{~S}_{6}$ with $P 6_{3} 22$ symmetry.

\section{Results and discussion}

Searching chiral materials from cation-intercalated TMDs

Intercalated TMDs have recently attracted substantial interest due to their intriguing properties arising from intercalation applications ${ }^{4-51}$. An important factor to be considered when intercalating a structure is that the host cannot be damaged significantly, such as a large-scale rearrangement of atoms or bonding distortions, which will remarkably increase the energy of the system and make the final structure unstable. The optimal solution to introduce chirality in layered TMDs could be adding screw rotation symmetry through the pivots within the vdW region, forming a chiral axis perpendicular to the vdW plane, which can guarantee minimal damage to the initial $\mathrm{MX}_{2}$ motifs, e.g., a triangular prism in the $2 \mathrm{H}$ phase and an octahedron in the $1 \mathrm{~T}$ phase. For simplicity, we consider cation intercalation in the $\sqrt{3} \times \sqrt{3} \times$ 2 supercell of the $1 \mathrm{~T}$ phase and the $\sqrt{3} \times \sqrt{3} \times 1$ supercell of the $2 \mathrm{H}$ phase with $\mathrm{AA}$ and $\mathrm{AB}$ stackings of $\mathrm{MX}_{2}$ layers. There are two types of possible intercalation patterns in each supercell with intercalated polyhedra: (i) vertically or (ii) nonvertically distributed along the $c$ axis (Figs. 1a, S1). Regarding the $\mathrm{AB}$ stacked $2 \mathrm{H}$ TMDs with type (ii) intercalation, there are two inequivalent configurations with the same space group (SG) $P 6_{3} 22$ (belonging to the 65 Sohncke groups that preserve chirality $)^{52}$ but opposite handedness in their intercalation patterns (see the circled red/blue spheres in Fig. 1a and the crystal structure in Fig. $\mathrm{S} 1$ ), in which the chiral symmetry is endowed by breaking the inversion and mirror symmetries of the pristine $A B$ stacked $2 \mathrm{H}$ phase $\left(P 6_{3} / \mathrm{mmc}\right)$. Regarding all the other intercalated situations, the products are achiral.

A series of new $\mathrm{AB}_{3} \mathrm{C}_{6}$ compounds can be obtained from the accessible TMD library ${ }^{53}$ with intercalated TM cations by the requirement of valence state matching $\left(X^{A}+3 X^{B}=6 X^{C}\right.$, assuming chalcogen with -2 common valence states) and inequivalent species on $\mathrm{I}(\mathrm{II}) / \mathrm{III}$ sites $(\mathrm{A} \neq \mathrm{B})$. All the $1 \mathrm{~T}$ intercalated TMDs present CS symmetry, namely, $P \overline{3} 1 \mathrm{~m}, P \overline{3} 1 \mathrm{c}, P 6_{3} / \mathrm{m}$, and $P 6_{3} / \mathrm{mcm}$. The $2 \mathrm{H}$ intercalated TMDs possess $1 \mathrm{CS}\left(\mathrm{Pb}_{3} / \mathrm{mcm}\right), 1$ chiral $\left(P 6_{3} 22\right)$, and 2 non-CS $(P \overline{6} 2 m$ and $P \overline{6} 2 c)$ structures (see Figs. $1 \mathrm{~b}, \mathrm{~S} 1)$. We evaluated the relative stability of the 
intercalated TMD structures and noted that (i) the intercalated cations are in octahedral (triangular prism) motifs for AA stacked $1 \mathrm{~T}$ and $\mathrm{AB}$ stacked $2 \mathrm{H}$ (AB stacked $1 \mathrm{~T}$ and $\mathrm{AA}$ stacked $2 \mathrm{H}$ ) phases; (ii) the intercalated octahedra tend to be nonvertically, rather than vertically, distributed along the $c$ axis, as in the low-energy $P \overline{3} 1 c$ and $P 6_{3} 22$ structures (Fig. S1). Such a nonvertical distribution of intercalated octahedra in the AA stacked $1 \mathrm{~T}$ layers of pristine octahedra pertains to CS symmetry, whereas the nonvertical distribution of octahedra intercalated between the $\mathrm{AB}$ stacked $2 \mathrm{H}$ layers of triangular prisms leads to chiral symmetry $\left(P 6_{3} 22\right)$. We demonstrated the origin of how chirality is induced within the system (Figs. 1c, S2).

A number of chiral TMD materials $\left(\mathrm{P6}_{3} 22\right)$ are magnetic, including those that were synthesized in experiments, such as $\mathrm{CoNb}_{3} \mathrm{~S}_{6}, \quad \mathrm{FeNb}_{3} \mathrm{~S}_{6}$, and $\mathrm{MnTa}_{3} \mathrm{~S}_{6}{ }^{54-58}$, and many others are nonmagnetic according to our DFT calculations (Fig. S3). We find a unique double hourglass electronic structure along the $\Gamma-\mathrm{A} k$-path around $\mathrm{E}_{\mathrm{F}}$ for the nonmagnetic chiral TMDs (Fig. S4), as the unshelled $d$ orbitals of their intercalated and/or host TM cations form partially occupied bands. In contrast, the double hourglass bands can be destroyed more or less in the magnetic case due to the broken time-reversal symmetry $(\mathcal{T}$ ) (Fig. S5). Therefore, we focus our research on the nonmagnetic chiral TMD for studying the potential chiralityassociated double hourglass fermion (Fig. 2a). Through careful examination of the electronic state and taking into account the material stability, we screened the best chiral TMD candidate, $\mathrm{RhV}_{3} \mathrm{~S}_{6}\left(P 6_{3} 22\right)$. The most stable $\mathrm{Rh}$ intercalated positions are $\mathrm{I} / \mathrm{II}$ and $\mathrm{I}^{\prime} / \mathrm{II}^{\prime}$ sites confirmed by globally detecting the total energy of the system when moving the cations in the vdW planes (Fig. S6). Further structural screening showed that $\mathrm{RhV}_{3} \mathrm{~S}_{6}$ in other known $\mathrm{AB}_{3} \mathrm{C}_{6}$ structure types had higher energy than the $P 6_{3} 22$ phase (Fig. 2b). The stability criteria in Fig. $2 \mathrm{c}$, d show that $\mathrm{RhV}_{3} \mathrm{~S}_{6}\left(P 6_{3} 22\right)$ is dynamically stable (no occurrence of virtual frequencies in its phonon spectrum) and has a relatively large stability region $\left(0.038 \mathrm{eV}^{2}\right)$ with respect to its competing phases. Thus, all the stability criteria prove the stability of $\mathrm{RhV}_{3} \mathrm{~S}_{6}$, indicating the feasibility of experimental synthesis.

\section{Double hourglass Weyl fermion in chiral TMD}

The chiral TMD $\left(\mathrm{RhV}_{3} \mathrm{~S}_{6}\right)$ crystallizes in a hexagonal structure with SG $D_{6}^{6}\left(\mathrm{PG}_{3} 22\right.$, No. 182), which presents semimetallic properties with valence and conduction bands crossing linearly at $(0,0, \pm 0.447 \pi)$ along $\Gamma-\mathrm{A}$ without the SOC effect (Fig. 3a, b) and forms a fourfold degenerated hourglass Dirac semimetal state (Fig. S7 and Table S2). In the presence of SOC, the energy bands are generally nondegenerate, except at the time-reversal invariant momenta (TRIM), e.g., $\Gamma$ and $A$, and the hourglass bands split into two nested hourglass bands (Fig. 3c, d). Here, we provide a symmetry argument for the hourglass dispersions along $\Gamma-\mathrm{A}$. The chiral TMD $\left(\mathrm{RhV}_{3} \mathrm{~S}_{6}\right)$ belongs to the nonsymmorphic space group $P 6_{3} 22$, which contains a screw rotation operator

$$
\tilde{C}_{2 z}(x, y, z) \rightarrow\left(-x,-y, z+\frac{1}{2}\right)
$$

where the tilde denotes a nonsymmorphic operation. The intercalated cations lead to chirality in the chiral TMDs $\left(P 6_{3} 22\right)$, whereas the nonsymmorphic $\tilde{C}_{2 z}$ symmetry is inherited from the parent $2 \mathrm{H}$ phase $\left(P 6_{3} / \mathrm{mmc}\right)$, leading to hourglass Weyl fermions. In addition, $\mathcal{T}$ symmetry is preserved for nonmagnetic $\operatorname{RhV}_{3} \mathrm{~S}_{6}$. The $\mathcal{T}$ symmetry guarantees Kramers degeneracies at TRIM $\Gamma$ and A due to $\mathcal{T}^{2}=-1$ in the presence of SOC. In the $\tilde{C}_{2 z}$ invariant $k$-path along $\Gamma-\mathrm{A}$, we can choose each Bloch state $|u\rangle$ to be the eigenstate of $\tilde{C}_{2 z}$. It is easily obtained that

$$
\tilde{C}_{2 z}^{2}|u\rangle=e^{-i k_{z}}|u\rangle,
$$

which indicates that $e^{-i k_{z}}$ is the eigenvalue of $\tilde{C}_{2 z}^{2}$. Thus, $\tilde{C}_{2 z}$ acts on the real-space part of the wavefunction and commutes with the time-reversal operator. Along the $\Gamma-\mathrm{A} k$ line, each $k$ point is invariant under $\tilde{C}_{2 z}$, and the related Bloch state $|u\rangle$ can be chosen as the eigenstates of $\tilde{C}_{2 z}$, namely, $\tilde{C}_{2 z}|u\rangle=g_{z}|u\rangle$. One has $\tilde{C}_{2 z}^{2}=e^{-i k_{z} / 2}$, and the eigenvalue of $\tilde{C}_{2 z}$ will be $g_{z}= \pm e^{-i k_{z} / 2}$, i.e., $g_{z}= \pm 1$ at the $\Gamma$ point $(0,0,0)$ and $g_{z}= \pm i$ at the point $(0,0, \pi)$. Since $\tilde{C}_{2 z}$ always commutes with the time-reversal symmetry $\mathcal{T}=K$ (without $\mathrm{SOC}$ ) or $\mathcal{T}=i \sigma y K$ (with SOC), where $K$ is the complex conjugate operator, the two Kramers pair $|u\rangle$ and $\mathcal{T}|u\rangle$ must degenerate at the time-reversal invariant points $\Gamma$ and $A$. In this case, the Kramers pair $|u\rangle$ and $\mathcal{T}|u\rangle$ at the $\Gamma$ point has the same eigenvalue, while the Kramers pair $|u\rangle$ and $\mathcal{T}|u\rangle$ at the A point have the opposite eigenvalue. Such partner switching between $\Gamma$ and $A$ enforces a band crossing with twofold degeneracy along $\Gamma-\mathrm{A}$. We further evaluated the double-valued representation of the double point group for each band and evidenced the state-switching characteristics, as seen from the degenerating and separating process of irreducible representations (irreps) at arbitrary point $\Delta$ along the $\Gamma-\mathrm{A}$ line for both chiral $\mathrm{S}$ and $\mathrm{R}$ phases in Fig. 3e and Table S3. Here, $\Delta_{i}$ is the irrep obtained by scanning each hourglass band along the $\Gamma-$ A line, forming a degenerated partner with another $\Delta_{j}$ at TRIM. The partners $\Delta_{11}$ and $\Delta_{8}\left(\Delta_{10}\right.$ and $\left.\Delta_{9}\right)$ form the top left (bottom) corner of one hourglass band at $\Gamma$, separate from each other while leaving from the $\Gamma$ point and recombine at the A point by exchanging the degenerated pair and constituting the right corners (Fig. S8). A similar 
(a)

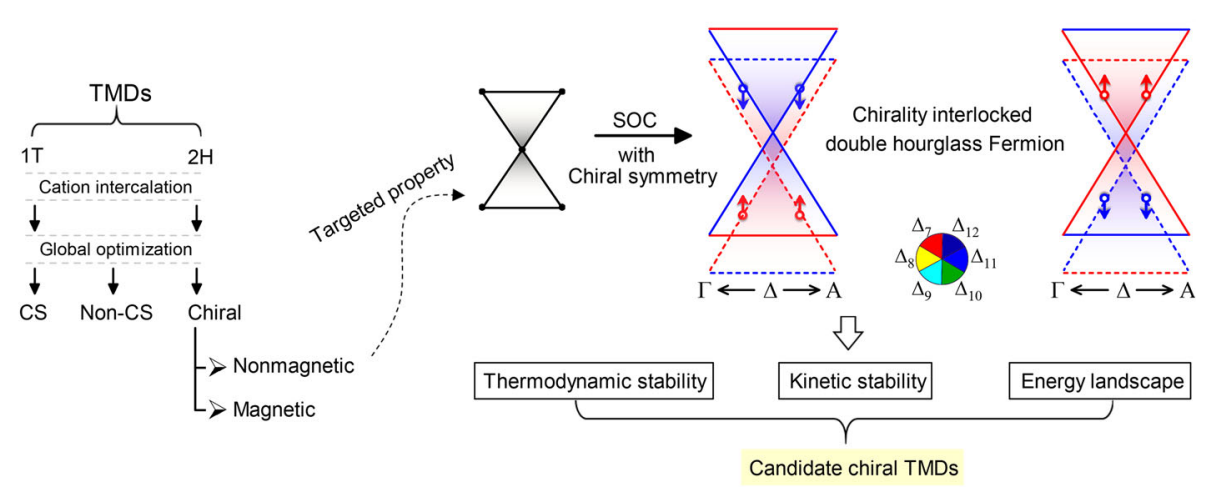

(b)

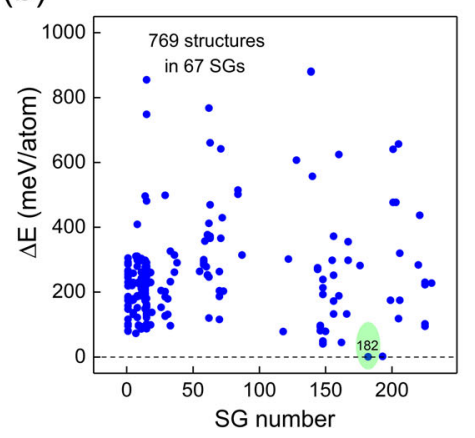

(c)

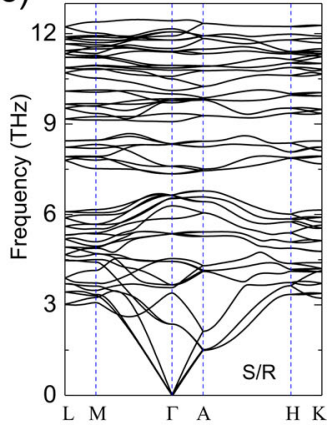

(d)

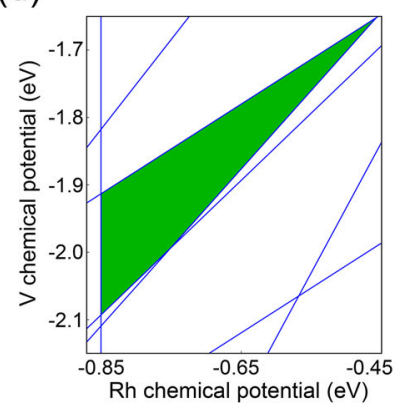

Fig. 2 Screening process and stability evaluation for stable chiral TMDs. a Design framework for the search of chiral TMDs with intercalated TM cations, targeting properties including explicit double hourglass fermions characterized by spin flipping between chiral structures with opposite handedness and structural stability. The potential chiral TMDs are divided into nonmagnetic and magnetic structures based on total energy evaluation. Subsequent electronic characteristic screening is performed for nonmagnetic structures with time-reversal symmetry. The stability of the screened chiral TMD was further evaluated by systematic material stability calculations. b Structural screening of $\mathrm{RhV}_{3} \mathrm{~S}_{6}$ by comparison with all possible phases possessing the same stoichiometry (A:B:C =1:3:6) distributed in 67 SGs. c Kinetic stability and (d) thermodynamic judgment for $\mathrm{RhV}_{3} \mathrm{~S}_{6}$. The nonimaginary frequency phonon dispersion suggests the most stable ground state for the chiral phase.

situation also holds for the other nested hourglass band formed by $\Delta_{7}$ and $\Delta_{12}\left(\Delta_{11}\right.$ and $\left.\Delta_{12}\right)$ degenerated partners. As a result, the occurrence of partner switching while moving from one TRIM to the other on the $\Gamma-\mathrm{A}$ line leads to the nontrivial band connectivity diagram and the nested double hourglass bands in $\mathrm{RhV}_{3} \mathrm{~S}_{6}$. Furthermore, we note that the spin characteristics for every hourglass band are completely opposite between chiral-S/R structures for the chiral TMDs, which indicates the interlocking of spin-momentum of the double hourglass bands and crystalline chirality,

$$
\psi(r, \uparrow) \stackrel{S / R}{\longleftrightarrow} \psi(r, \downarrow) .
$$

Here, the $r$ stands for spatial coordinates. It should be noted that the linear energy dispersion of the double hourglass bands extends over a relatively large energy range and thus could be experimentally observed uncomplicatedly via various spectroscopy techniques (e.g., tunneling spectroscopy, photoemission, or transport experiments) ${ }^{32,59}$.

\section{Tuning the dia-chiral response and double hourglass state} by strain

The interlocking of crystalline chirality and double hourglass bands indicates the possibility of tuning one through another. The left-/right-handed $\mathrm{RhV}_{3} \mathrm{~S}_{6}$ configurations are determined by the placement of Rh octahedra (at I/II and $\mathrm{I}^{\prime} / \mathrm{II}^{\prime}$ sites) and present opposite $\mathrm{CD}$ responses (Fig. 4). The octahedron in pristine $\mathrm{RhV}_{3} \mathrm{~S}_{6}$ maintains a slight atomic distortion for the $\mathrm{V}-1$ and $\mathrm{S}$ atoms: the $\mathrm{V}-1$ atoms that are originally located on the $a b$ plane have relative displacements along the $c$ axis $\left(d_{\mathrm{V}-1}=\right.$ $0.0015 \AA)$; the $\mathrm{S}$ atoms rotate in the $a b$ plane $\left(0.594^{\circ} /\right.$ $0.297^{\circ}$ for $\alpha$ and $\beta$, respectively). In general, the local atomic distribution and bonding environment could affect the chirality of the system. The enhanced distorting operation (e.g., rotation) is usually considered the origin of chirality or the main contributor ${ }^{60-62}$. However, not all atomic bonding distortions contribute to the chirality, such as the Kekule distortion ${ }^{63}$ for epitaxial graphene on a copper substrate that breaks the chiral symmetry ${ }^{64}$. Since distortion can increase or decrease chirality, one fundamental question is whether an intrinsic chirality hidden in 


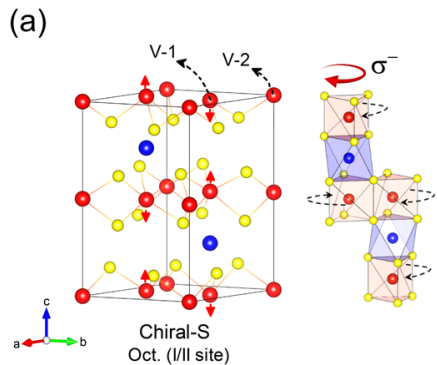

(b)

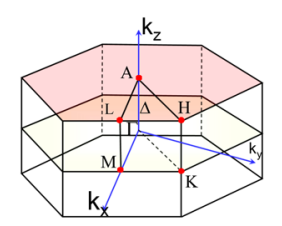

(d)

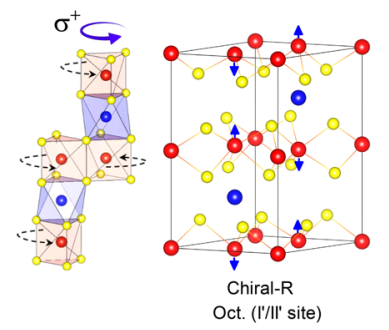

(e)

(c)
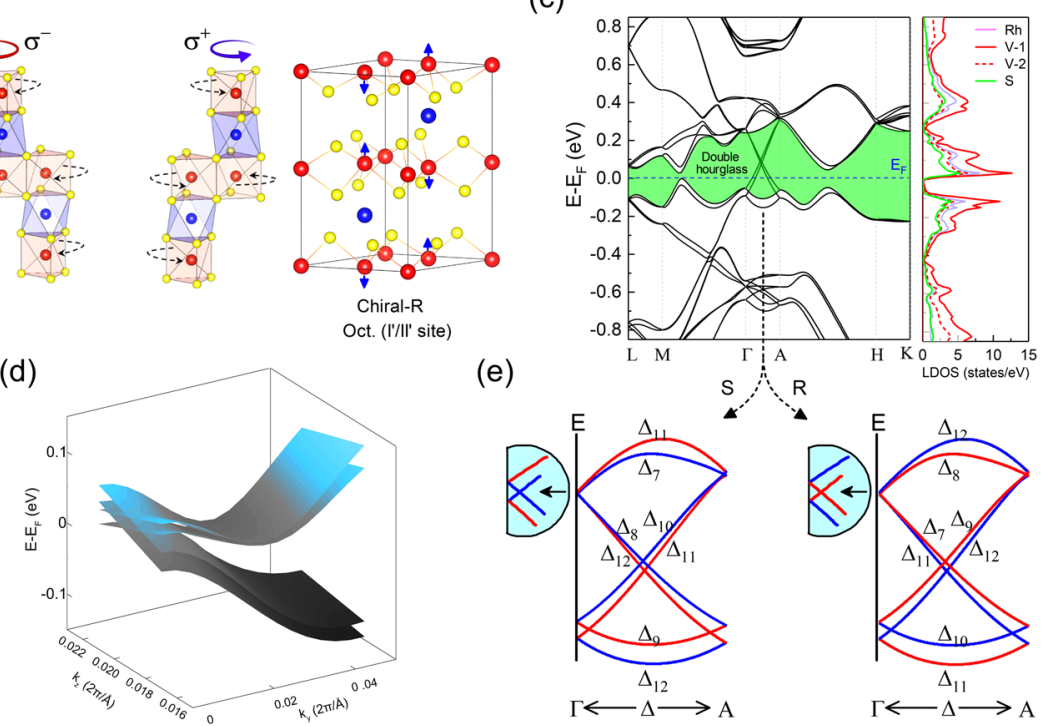

(e)

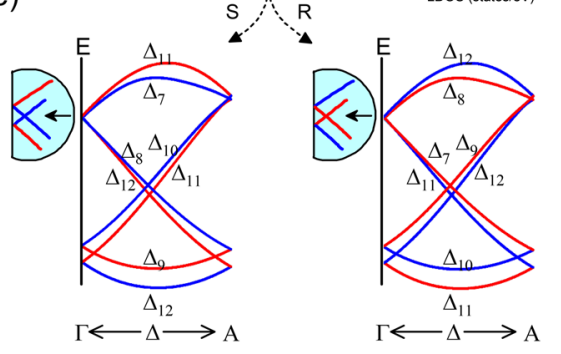

Fig. 3 Double hourglass Weyl fermion state in $\mathbf{R h V}_{\mathbf{3}} \mathbf{S}_{\mathbf{6}}$. a Crystal structure of $\mathrm{RhV}_{3} \mathrm{~S}_{6} \mathbf{W i t h} \mathbf{P}_{3} 22$ symmetry, in which the left- and right-handed configurations of the intercalated TMDs are indicated by $S$ and $\mathrm{R}$ with cations positioned at $I / I I\{(2 / 3,1 / 3,3 / 4) /(1 / 3,2 / 3,1 / 4)\}$ and $I^{\prime} / I^{\prime}\{(2 / 3,1 / 3,1 / 4) /$ $(1 / 3,2 / 3,3 / 4)\}$ sites of the crystal. The two inequivalent $V$ sites are denoted as $V-1$ and $V-2$. The $V-1 S_{6}$ triangular prisms (in red) directly connected with the Rh octahedra (in blue) are twisted oppositely in the two chiral structures. b Brillouin zone for the hexagonal structure with symmetry labels for high-symmetry points. $\mathbf{c}$ Band structure of $\mathrm{RhV}_{3} \mathrm{~S}_{6}\left(\mathrm{P}_{3} 22\right)$ with $\mathrm{SOC}$ and the local density of states (LDOS) for different species of atoms in the primitive cell. $\mathbf{d}$ 3D band plotting of the double hourglass fermions. e Double hourglass bands with irreducible representations for each band in chiral-S/R configurations, where the red/blue colors indicate the spin polarizations (along the z-axis).

a specific microstructure exists and whether local atomic distortion will force it to decay. Here, we probed this issue in our predicted chiral TMD $-\mathrm{RhV}_{3} \mathrm{~S}_{6}$, as the inherent chirality is intimated with the special configuration of the intercalated octahedra (Fig. 1c) and can be sensitively captured by the double hourglass fermion by controlling the intercalation-induced local structure distortions. This is done by applying biaxial strains ( $a b$ in-plane) on $\mathrm{RhV}_{3} \mathrm{~S}_{6}$. We note that as the perturbation is induced via strain, the relative movement of $\mathrm{V}-1$ diminishes gradually, whereas the rotation angle of $\mathrm{S}$ experiences a certain degree of fluctuation (Fig. 4b). Both of these structural deformation factors reach the critical point around the $+2.5 \%$ strained case, as the octahedron recovers to its pristine structure with barely any rotation. Additionally, the $\mathrm{CD}$ intensity presents the maximum for the pristine octahedron stacking configuration, while both the enhanced perturbation of $\mathrm{S}(\alpha$ and $\beta)$ and $\mathrm{V}-1\left(d_{\mathrm{V}-}\right.$ $\left.{ }_{1}\right)$ distortions weaken the CD signal (Fig. 4c). Even for the slightly distorted octahedra in unstrained $\mathrm{RhV}_{3} \mathrm{~S}_{6}$, the CD response is weakened. To further demonstrate the intrinsic chirality restored within intercalated octahedra, we separately evaluated the CD response under $+2.5 \%$ strain with increasing $\mathrm{S}$ (Rot. $\mathrm{S} ; \alpha, \beta$ ) and V -1 (Dist. V $\left.1 ; d_{\mathrm{V}-1}\right)$ distortions, starting from the structure with quenched atomic distortion, which indicates the compensation of intrinsic chirality (for quenched distortion, see the curves with the highest $\mathrm{CD}$ peaks in Fig. $4 \mathrm{~d}$ ). Indeed, the intrinsic chirality of $\mathrm{RhV}_{3} \mathrm{~S}_{6}$ is introduced by the pristine octahedron placement configuration, forming the intrinsic chiral domain of the crystal. However, the domain steady state will be broken by structural perturbation due to redistributed state coupling (i.e., rebalancing of the wavefunction), leading to the opposite handedness and weakening of the intrinsic chirality, as an inevitable "dia-chiral response" that can be modulated by external strain, as discussed above.

In the "dia-chiral response" modulation, we find that the double hourglass state presents a tunable hourglass window, namely, the covered spin-splitting energy range in $E-k$ space by hourglass bands. The splitting window for pristine $\mathrm{RhV}_{3} \mathrm{~S}_{6}$ reaches a record energy range of $\sim 380 \mathrm{meV}$ (Fig. 3c), which is significantly larger than those in $\mathrm{KHgSb}(60 / 80 \mathrm{meV}$ for $\tilde{\mathrm{Z}} / \tilde{\mathrm{X}}-\tilde{\Gamma} / \tilde{\mathrm{U}}$ at 010 -surface $)^{22}, \mathrm{Ag}_{2} \mathrm{BiO}_{3}(3 \mathrm{meV} \text { for } \mathrm{X}-\mathrm{S} \mathrm{k} \text { line })^{27}$, and $\mathrm{Bi} /$ $\mathrm{Cl}-\mathrm{SiC}$ with a 111 -plane $(150 / 187 \mathrm{meV}$ for $\mathrm{X} / \Gamma-\mathrm{M} / \mathrm{Y}$ direction). Strain engineering enables the wide-range modulation of the double hourglass bands $(\sim 300-440 \mathrm{meV})$ for $\mathrm{RhV}_{3} \mathrm{~S}_{6}$ (Fig. S9). In the $+2.5 \%$ strained case with the maximum CD intensity, the middle band-crossing points (the normal centers of the double hourglass bands; analogous to the Weyl points in achiral TMD Weyl semimetals ${ }^{13-17}$ ) shift to the $E_{F}$ (Fig. 4e). In fact, the double hourglass bands are dominated by V $3 d$ 
(a)



(b)

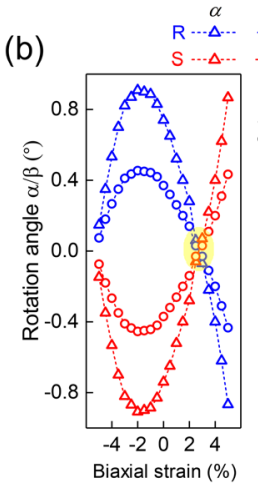

$$
\begin{array}{ccc}
\alpha & \beta & d_{\mathrm{V}-1} \\
\mathrm{R} \cdots \Delta \cdots & \cdots 0 & \cdots \square
\end{array}
$$

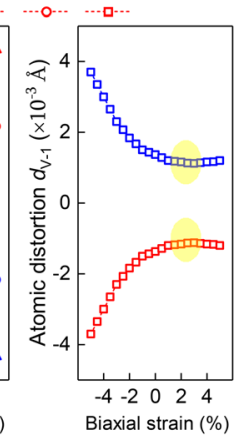

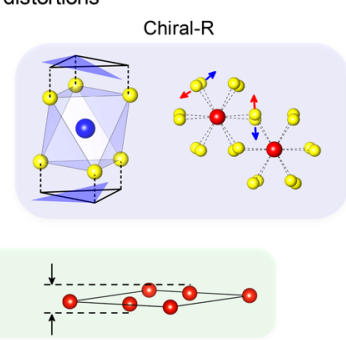

(c)

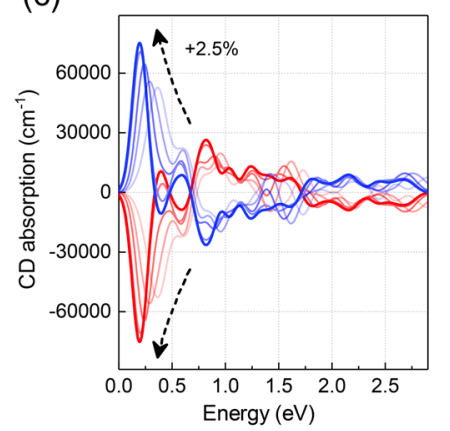

(e)

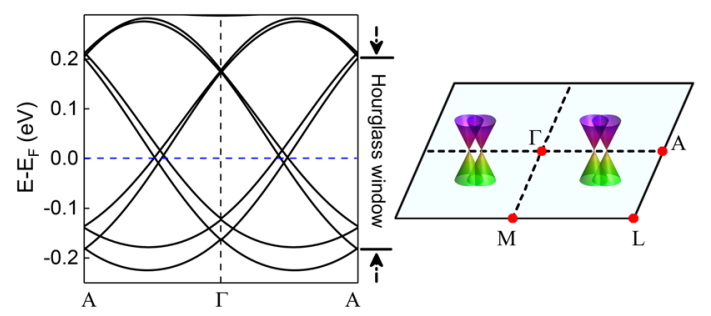

(d)

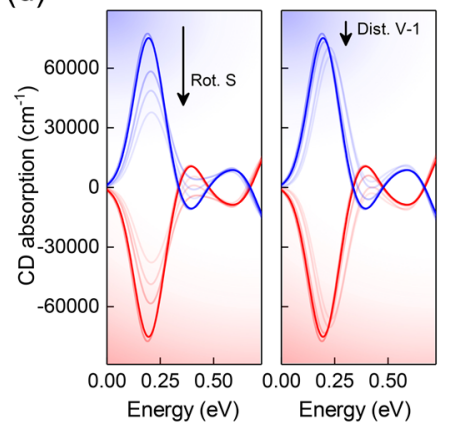

Fig. 4 Chirality modulation and dia-chiral response in $\mathrm{RhV}_{3} \mathrm{~S}_{6}$. a Schematic diagram of local environmental distortions, including the planar rotation $\left(a\right.$ and $\beta$ ) of $S$ atoms and relative vertical movement of $V-1\left(d_{V-1}\right)$ atoms. $\mathbf{b}$ Local environment distortions as functions of biaxial strain. c Evolution of the $C D$ spectrum under $a b$ in-plane biaxial strain for $\mathrm{RhV}_{3} \mathrm{~S}_{6}$. The strongest $C D$ response of $\mathrm{RhV}_{3} \mathrm{~S}_{6}$ occurs at approximately $+2.5 \%$ strain with the minimum local environment distortion ( $a, \beta$ less than $0.08^{\circ} / 0.04^{\circ}$ and $d_{V-1}<10^{-3} \AA$ ). $\mathbf{d}$ Evolution of the CD spectrum under $+2.5 \%$ ab in-plane biaxial strain from completely quenched distortion to gradually increased $\mathrm{S}$ rotation $\left(\mathrm{V}-1\right.$ distortion) imposed on $\mathrm{RhV}_{3} \mathrm{~S}_{6}$. e Ideal double hourglass bands for $\mathrm{RhV}_{3} \mathrm{~S}_{6}(+2.5 \%$ strained case) with a spin-splitting energy window of $\sim 355 \mathrm{meV}$.

orbitals hybridized with $\mathrm{Rh} 4 d$ and $\mathrm{S} 3 p$ components, forming anti-bonding states (Figs. 3c, S10, S11). When $a b$ in-plane tensile strain is applied, the heights $\left(h_{\text {Oct. }}\right.$ and $h_{\text {Tri. }}$ ) of the octahedron and triangular prism are both compressed (Fig. S11), and the Rh-S and V-S bond lengths $\left(d_{\mathrm{Rhs}}\right.$. and $\left.d_{\mathrm{VS}}\right)$ are enlarged. As a consequence, the $p$ - $d$ coupling between the $\mathrm{V} 3 d$ (or $\mathrm{Rh} 4 d$ ) and S $3 p$ orbitals is weakened due to the decreased overlap between their wavefunctions, which tends to lower the anti-bonding double hourglass states. Therefore, the double hourglass bands continuously move down with a decreasing splitting window, presenting flexible tunability along with the modulation of crystalline chirality.

\section{Conclusions}

In summary, we predict a number of octahedral intercalated chiral TMDs with a type of spin-split double hourglass Weyl fermion, which is interlocked with crystalline chirality. The optical CD of intercalated TMDs is evaluated to monitor the chirality of the material that can be tuned by external strain. The best candidate $\mathrm{RhV}_{3} \mathrm{~S}_{6}$ is predicted to be thermodynamically stable, whose synthesis is called for as the first example of a chirality-enforced double hourglass fermion. On the other hand, this type of chiral TMD may provide a new platform for exploring tunable chiraltronics within a three-dimensional scale, e.g., a chiral plasmonic device ${ }^{65-67}$, as three-dimensional chiral plasmonic structures that exhibit a strong intrinsic chiral optical response remain an extreme challenge. Furthermore, the predicted $\mathrm{AB}_{3} \mathrm{C}_{6}$ family members could also raise great interest in extraordinary phenomena and device applications, such as the anomalous Hall effect ${ }^{54}$, mesoscopic magnetic modulation ${ }^{68}$, three-state nematicity $^{69}$, and tunable giant exchange bias by spin glass ${ }^{70}$. The stable properties and facile synthesis of these chiral TMDs guarantee the subsequent micro/nanoscale process and device integration. Our study provides an in-depth mechanism of intrinsic chirality induction through polyhedral intercalation in TMDs, which have a significant chiral optical response. More importantly, insight into introducing intrinsic chirality through chiral intercalation of polyhedra in achiral systems to realize a strong chiral response will shed light on designing/searching for new chiral structures extensively with novel characteristics. Our findings are one step forward in the exploration of chirality-related hourglass Weyl fermions in inorganic compounds and provide a new understanding of structural chirality for future chiral-functionalized optoelectronic devices. 


\section{Acknowledgements}

The authors would like to thank Prof. Lin-Wang Wang for helpful suggestions. This work is supported by the National Natural Science Foundation of China (Grant Nos. 11804230, 11774239, 61827815), Shenzhen Science and Technology Innovation Commission (Grant Nos. KQTD20180412181422399, KQTD20170810105439418, JCYJ20170818093035338), National Key R\&D Program of China (Grant No. 2016YFB0700700), High-Level University Construction Funds of SZU (Grant No. 860-000002081209 and 860000002110711 ) and Fundamental Research Funds for the Central Universities (No. 2018CDXYWU0025)

\section{Author details}

'Key Laboratory of Optoelectronic Devices and Systems of Ministry of Education and Guangdong Province, College of Physics and Optoelectronic Engineering, Shenzhen University, 518060 Shenzhen, China. ${ }^{2}$ Shenzhen Institute for Quantum Science and Technology and Department of Physics, Southern University of Science and Technology (SUSTech), 518055 Shenzhen, China. ${ }^{3}$ Department of Physics, Chongqing University, 401331 Chongqing, China. ${ }^{4}$ Collaborative Innovation Center for Optoelectronic Science \& Technology, Key Laboratory of Optoelectronic Devices and Systems of Ministry of Education and Guangdong Province, College of Physics and Optoelectronic Engineering, Shenzhen University, 518060 Shenzhen, China

\section{Author contributions}

X.Z. directed the research project. P.H. performed the calculations and prepared the figures. P.H., Q.L., R.W., H.S., and X.Z. conducted the symmetry analysis of the chiral TMDs. X.C., X.Z., and H.Z. evaluated the stability of these compounds. P.Z., S.X., and W.X. analyzed the state coupling and spin characteristics of $\mathrm{RhV}_{3} \mathrm{~S}_{6}$. All authors contributed to the discussion and provided inputs to the paper. P.H., X.C., and P.Z. contributed equally to this work.

\section{Competing interests}

The authors declare no competing interests.

\section{Publisher's note}

Springer Nature remains neutral with regard to jurisdictional claims in published maps and institutional affiliations.

Supplementary information The online version contains supplementary material available at https://doi.org/10.1038/s41427-021-00316-w.

Received: 10 November 2020 Revised: 21 April 2021 Accepted: 24 May 2021.

Published online: 25 June 2021

\section{References}

1. Mak, K. F., Lee, C., Hone, J., Shan, J. \& Heinz, T. F. Atomically thin MoSz: a new direct-gap semiconductor. Phys. Rev. Lett. 105, 136805 (2010).

2. Mak, K. F. et al. Tightly bound trions in monolayer $\mathrm{MoS}_{2}$. Nat. Mater. 12 , 207-211 (2013).

3. Chernikov, A. et al. Exciton binding energy and nonhydrogenic Rydberg series in monolayer WS 2 . Phys. Rev. Lett. 113, 076802 (2014).

4. Cao, T. et al. Valley-selective circular dichroism of monolayer molybdenum disulphide. Nat. Commun. 3, 887 (2012).

5. Wang, G. et al. Control of exciton valley coherence in transition metal dichalcogenide monolayers. Phys. Rev. Lett. 117, 187401 (2016).

6. Schaibley, J. R. et al. Valleytronics in $2 \mathrm{D}$ materials. Nat. Rev. Mater. 1, 16055 (2016).

7. Zhang, L. \& Niu, Q. Chiral phonons at high-symmetry points in monolayer hexagonal lattices. Phys. Rev. Lett. 115, 115502 (2015).

8. Li, Z. et al. Tailoring $\mathrm{MoS}_{2}$ valley-polarized photoluminescence with super chiral near-field. Adv. Mater. 30, 1801908 (2018).

9. Gong, S.H., Alpeggiani, F., Sciacca, B., Garnett, E. C. \& Kuipers, L. Nanoscale chiral valley-photon interface through optical spin-orbit coupling. Science $\mathbf{3 5 9}$ 443-447 (2018).
10. Chery, T. et al. Room temperature chiral coupling of valley excitons with spinmomentum locked surface plasmons. ACS Photonics 5, 1281-1287 (2018).

11. Zhang, H., He, H., Jiang, X., Xia, Z. \& Wei, W. Preparation and characterization of chiral transition-metal dichalcogenide quantum dots and their enantioselective catalysis. ACS Appl. Mater. Inter. 10, 30680-30688 (2018).

12. Purcell-Milton, F. et al. Induction of chirality in two-dimensional nanomaterials: chiral 2D MoS 2 nanostructures. ACS Nano 12, 954-964 (2018).

13. Deng, K. et al. Experimental observation of topological Fermi arcs in type-ll Weyl semimetal MoTe 2 . Nat. Phys. 12, 1105-1110 (2016).

14. Soluyanov, A. A. et al. Type-ii weyl semimetals. Nature 527, 495-498 (2015).

15. Sun, Y., Wu, S.-C., Ali, M. N., Felser, C. \& Yan, B. Prediction of Weyl semimetal in orthorhombic MoTe2. Phys. Rev. B 92, 161107 (2015).

16. Jiang, J. et al. Signature of type-Il Weyl semimetal phase in $\mathrm{MoTe}_{2}$. Nat. Commun. 8, 13973 (2017).

17. Li, P. et al. Evidence for topological type-ll Weyl semimetal WTe 2 . Nat. Commun. 8, 1-8 (2017).

18. Ali, M. N. et al. Large, non-saturating magnetoresistance in $\mathrm{WTe}_{2}$. Nature $\mathbf{5 1 4}$, 205-208 (2014)

19. Chen, F. C. et al. Extremely large magnetoresistance in the type-ll Weyl semimetal $\mathrm{MoTe}_{2}$. Phys. Rev. B 94, 235154 (2016).

20. Chang, G. et al. Topological quantum properties of chiral crystals. Nat. Mater. 17, 978-985 (2018).

21. Young, S. M. \& Kane, C. L. Dirac semimetals in two dimensions. Phys. Rev. Lett. 115, 126803 (2015)

22. Wang, Z., Alexandradinata, A., Cava, R. J. \& Bernevig, B. A. Hourglass fermions. Nature 532, 189-194 (2016).

23. Wang, L., Jian, S.-K. \& Yao, H. Hourglass semimetals with nonsymmorphic symmetries in three dimensions. Phys. Rev. B 96, 075110 (2017).

24. Chen, Y., Kim, H.S. \& Kee, H.-Y. Topological crystalline semimetals in nonsymmorphic lattices. Phys. Rev. B 93, 155140 (2016).

25. Wang, S.-S., Liu, Y., Yu, Z.-M., Sheng, X.L. \& Yang, S. A. Hourglass Dirac chain metal in rhenium dioxide. Nat. Commun. 8, 1844 (2017).

26. Takahashi, R., Hirayama, M. \& Murakami, S. Spinless hourglass nodal-line semimetals. Phys. Rev. B 96, 155206 (2017).

27. Singh, B. et al. Topological hourglass dirac semimetal in the nonpolar phase of $\mathrm{Ag}_{2} \mathrm{BiO}_{3}$. Phys. Rev. Lett. 121, 226401 (2018).

28. Fu, B., Fan, X., Ma, D., Liu, C.C. \& Yao, Y. Hourglasslike nodal net semimetal in $\mathrm{Ag}_{2} \mathrm{BiO}_{3}$. Phys. Rev. B 98, 075146 (2018).

29. Shao, D. et al. Composite topological nodal lines penetrating the Brillouin zone in orthorhombic AgF $F_{2}$. NPJ Comput. Mater. 5, 53 (2019).

30. Zeng, Y., Wang, L. \& Yao, D.-X. n-Hourglass Weyl fermions in nonsymmorphic materials. Phys. Rev. B 101, 115110 (2020).

31. Wang, Z. F., Liu, B. \& Zhu, W. Hourglass fermion in two-dimensional material. Phys. Rev. Lett. 123, 126403 (2019).

32. Ma, J. et al. Experimental evidence of hourglass fermion in the candidate nonsymmorphic topological insulator KHgSb. Sci. Adv. 3, e1602415 (2017).

33. Kresse, G. \& Joubert, D. From ultrasoft pseudopotentials to the projector augmented-wave method. Phys. Rev. B 59, 1758 (1999).

34. Perdew, J. P., Burke, K. \& Ernzerhof, M. Generalized gradient approximation made simple. Phys. Rev. Lett. 77, 3865 (1996).

35. Kohn, W. \& Sham, L. J. Self-consistent equations including exchange and correlation Effects. Phys. Rev. 140, A1133 (1965).

36. Kresse, G. \& Furthmüller, J. Efficiency of ab-initio total energy calculations for metals and semiconductors using a plane-wave basis set. Comput. Mater. Sci. 6, 15-50 (1996)

37. Monkhorst, H. J. \& Pack, J. D. Special points for Brillouin-zone integrations. Phys. Rev. B 13, 5188 (1976).

38. Heyd, J., Scuseria, G. E. \& Ernzerhof, M. Erratum: "Hybrid functionals based on a screened Coulomb potential" [J. Chem. Phys.118, 8207 (2003)]. J. Chem. Phys. 124, 219906 (2006).

39. Heyd, J., Scuseria, G. E. \& Ernzerhof, M. Hybrid functionals based on a screened Coulomb potential. J. Chem. Phys. 118, 8207-8215 (2003).

40. Ehrenreich, H. \& Cohen, M. H. Self-consistent field approach to the manyelectron problem. Phys. Rev. 115, 786 (1959).

41. Bohm, D. \& Pines, D. A collective description of electron interactions: III. Coulomb interactions in a degenerate electron gas. Phys. Rev. 92, 609 (1953).

42. Gajdoš, M., Hummer, K, Kresse, G., Furthmüller, J. \& Bechstedt, F. Linear optical properties in the projector-augmented wave methodology. Phys. Rev. B 73, 045112 (2006).

43. Jia, W. et al. The analysis of a plane wave pseudopotential density functional theory code on a GPU machine. Comput. Phys. Commun. 184, 9-18 (2013). 
44. Jia, W. et al. Fast plane wave density functional theory molecular dynamics calculations on multi-GPU machines. J. Comput. Phys. 251, 102-115 (2013).

45. Bergerhoff, G., Brown, I. D. \& Allen, F. Crystallographic Databases. International Union of Crystallography, Chester (1987).

46. Jain, A. et al. Commentary: the materials project: a materials genome approach to accelerating materials innovation. APL Mater. 1, 011002 (2013).

47. Zhang, X., Yu, L., Zakutayev, A. \& Zunger, A. Sorting stable versus unstable hypothetical compounds: the case of multi-functional ABX half-heusler filled tetrahedral structures. Adv. Funct. Mater. 22, 1425-1435 (2012).

48. Togo, A., Oba, F. \& Tanaka, I. First-principles calculations of the ferroelastic transition between rutile-type and $\mathrm{CaCl}_{2}$-type $\mathrm{SiO}_{2}$ at high pressures. Phys. Rev. B 78, 134106 (2008)

49. Nair, N. L. et al. Electrical switching in a magnetically intercalated transition metal dichalcogenide. Nat. Mater. 19, 153-157 (2020).

50. Liu, X.-C. et al. Spontaneous self-intercalation of copper atoms into transition metal dichalcogenides. Sci. Adv. 6, eaay4092 (2020).

51. Inoshita, T., Hirayama, M., Hamada, N., Hosono, H. \& Murakami, S. Topological semimetal phases manifested in transition metal dichalcogenides intercalated with 3d metals. Phys. Rev. B 100, 121112 (2019).

52. Hiller, H. Crystallography and cohomology of groups. Am. Math. Mon. 93, 765-779 (1986).

53. Zhou, J. et al. A library of atomically thin metal chalcogenides. Nature $\mathbf{5 5 6}$ 355-359 (2018).

54. Ghimire, N. J. et al. Large anomalous Hall effect in the chiral-lattice antiferromagnet $\mathrm{CoNb}_{3} \mathrm{~S}_{6}$. Nat. Commun. 9, 3280 (2018).

55. Anzenhofer, K., Van Den Berg, J. M., Cossee, P. \& Helle, J. N. The crystal structure and magnetic susceptibilities of $\mathrm{MnNb}_{3} \mathrm{~S}_{6}, \mathrm{FeNb}_{3} \mathrm{~S}_{6}, \mathrm{CoNb}_{3} \mathrm{~S}_{6}$ and $\mathrm{NiNb}_{3} \mathrm{~S}_{6}$. J. Phys. Chem. Solids 31, 1057-1067 (1970).

56. Vaqueiro, P., Kosidowski, M. L. \& Powell, A. V. Structural distortions of the metal dichalcogenide units in $\mathrm{AMo}_{2} \mathrm{~S}_{4}(\mathrm{~A}=\mathrm{V}, \mathrm{Cr}, \mathrm{Fe}, \mathrm{Co})$ and magnetic and electrical properties. Chem. Mater. 14, 1201-1209 (2002).
57. Ueno, T., Yamamoto, K., Matsukura, H., Kusawake, T. \& Ohshima, K. X-ray structural study of the layered compounds $\mathrm{Co}_{x} \mathrm{NbS}_{2}$. Sci. Technol. Adv. Mat. 6 684-688 (2005).

58. van den Berg, J. M. \& Cossee, P. Structural aspects and magnetic behaviour of $\mathrm{NbS}_{2}$ and $\mathrm{TaS}_{2}$ containing extra metal atoms of the first transition series. Inorg Chim. Acta 2, 143-148 (1968).

59. LV, B. Q. et al. Observation of three-component fermions in the topological semimetal molybdenum phosphide. Nature 546, 627-631 (2017).

60. Lipkowitz, K. B., Schefzick, S. \& Avnir, D. Enhancement of enantiomeric excess by ligand distortion. J. Am. Chem. Soc. 123, 6710-6711 (2001).

61. Baimuratov, A. S. et al. Optical anisotropy of topologically distorted semiconductor nanocrystals. Nano Lett. 17, 5514-5520 (2017).

62. Shafer, P. et al. Emergent chirality in the electric polarization texture of titanate superlattices. Proc. Natl Acad. Sci. U.S.A. 115, 915-920 (2018).

63. Hou, C.-Y., Chamon, C. \& Mudry, C. Electron fractionalization in twodimensional graphenelike structures. Phys. Rev. Lett. 98, 186809 (2007).

64. Gutiérrez, C. et al. Imaging chiral symmetry breaking from Kekulé bond order in graphene. Nat. Phys. 12, 950-958 (2016).

65. Gao, W. et al. Photonic Weyl degeneracies in magnetized plasma. Nat. Commun. 7, 12435 (2016).

66. Zyuzin, A. A., Silaev, M. \& Zyuzin, V. A. Nonlinear chiral transport in Dirac semimetals. Phys. Rev. B 98, 205149 (2018).

67. Hentschel, M. et al. Chiral plasmonics. Sci. Adv. 3, e1602735 (2017).

68. Karna, S. K. et al. Observation of a mesoscopic magnetic modulation in chiral $\mathrm{Mn}_{1 / 3} \mathrm{NbS}_{2}$. arXiv https://arxiv.org/abs/1905.00452 (2019).

69. Little, A. et al. Observation of three-state nematicity in the triangular lattice antiferromagnet $\mathrm{Fe}_{1 / 3} \mathrm{NbS}_{2}$. arXiv https://arxiv.org/abs/1908.00657 (2019).

70. Doyle, S. et al. Tunable giant exchange bias in an intercalated transition metal dichalcogenide. arXiv https://arxiv.org/abs/1904.05872 (2019). 